\title{
水稻几丁质酶基因的转录与表达特征
}

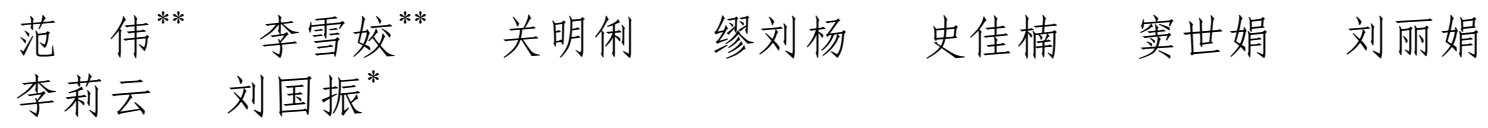

河北农业大学生命科学学院, 河北保定 071000

摘 要: 几丁质酶与植物的生长发育、抗逆和防御反应相关。水稻 PR3 家族几丁质酶编码基因有 19 个成员, 本文分 析其转录特征, 了解到有些基因属于组成型转录, 有些基因属于组织特异型转录, 分析几丁质酶蛋白质的结构域, 并对其进行聚类和亚家族分类。利用免疫印迹技术分析几丁质酶蛋白质的表达谱, 发现 CHIT5 的表达量在水稻叶片 生长过程中下调, 而 CHIT6、CHIT14、CHITC1 和 CHITC2 的表达量上调。在水稻与白叶枯病菌(Xoo)的不亲和反应 中, CHIT1、CHIT2、CHIT5、CHIT6、CHIT10、CHIT15 和 CHIT16 的表达量上调, CHIT14、CHITC1 和 CHITC2 的 表达下调。进一步比较几丁质酶蛋白质在水稻-Xoo 不同互作反应中的表达, 发现其在亲和及不亲和反应中的表达模 式类似，但一般在亲和反应中强度变化较大。此外, CHIT6 蛋白质在对照反应中的表达量上调, 提示 CHIT6 的表达受 创伤的诱导。本文比较系统地揭示了水稻 PR3 家族几丁质酶编码基因的转录和蛋白质表达特征, 为其功能解析提供 了线索。

关键词: 水稻; 几丁质酶; 聚类分析; 白叶枯病菌; 转录; 免疫印迹

\section{Transcriptional and Translational Characterization of Rice Chitinase Genes}

FAN Wei**, LI Xue-Jiao**, GUAN Ming-Li, MIAO Liu-Yang, SHI Jia-Nan, DOU Shi-Juan, LIU Li-Juan, LI Li-Yun, and LIU Guo-Zhen"

College of Life Sciences, Agricultural University of Hebei, Baoding 071000, China

\begin{abstract}
Plant chitinases play roles in plant development and stress responses. Nineteen chitinase genes, belonging to pathogenesis related PR3 family, were found in rice genome. In this study, constitutive and tissue-specific transcripted chitinase genes were identified, and the domain architecture of chitinase protein was predicted. Clustering analysis and subfamily classification were also carried out. Furthermore, chitinase protein expression profiling was surveyed using western blot (WB), it was found that the expression of CHIT5 was down-regulated in normal growth rice leaves, while the expressions of CHIT6, CHIT14, CHITC1, and CHITC2 were up-regulated. In the incompatible interaction between rice and Xanthomonas oryzae pv. oryzae (Xoo), the expressions of CHIT1, CHIT2, CHIT5, CHIT6, CHIT10, CHIT15, and CHIT16 were enhanced after inoculation and three of CHIT14, CHITC1 and CHITC2 down-regulated. In addition, the comparison of chitinase protein expression in different rice-Xoo interactions showed that these were similar alternation patterns between compatible and incompatible reactions, with a higher extent of alterations in incompatible interactions for most of chitinases. It is interesting to note that the expression of CHIT6 was enhanced in mock-treated samples, suggesting that the expression of CHIT6 may be induced by a mechanic wound. The data revealed in this research will provide useful clues for the understanding of the function of PR3 family chitinase genes.
\end{abstract}

Keywords: Rice; Chitinase; Clustering analysis; Xanthomonas oryzae pv. oryzae (Xoo); Transcription; Western blot

几丁质(chitin)又称壳多糖，其在甲壳类动物的 外壳、昆虫的甲壳和真菌的细胞壁以及绿藻中广泛
存在。几丁质是由 1000 3000 个 N-乙酰葡萄糖胺残 基通过 $\beta-1,4$ 糖苷键相互连接而成的聚合物, 其分子

\footnotetext{
本研究由国家自然科学基金项目(31171528)资助。

“通讯作者(Corresponding author): 刘国振, E-mail: gzhliu@genomics.org.cn, Tel: 0312-7528250

第一作者联系方式: E-mail: fanwei_mbb@126.com (范伟); lixuejiao_mbb@126.com (李雪姣), Tel: 0312-7528787

**同等贡献(Contributed equally to this work)

Received(收稿日期): 2013-08-08; Accepted(接受日期): 2014-01-12; Published online(网络出版日期): 2014-02-14.

URL: http://www.cnki.net/kcms/detail/11.1809.S.20140214.1018.007.html
} 
量往往在 $1000 \mathrm{kD}$ 以上。它在生物体中主要是作为 身体骨架且具自身保护作用。在植物与病原物的互 作过程中，几丁质的降解产物 N-乙酰葡萄糖胺可以 发挥病原物相关的分子模式(microbe/pathogen associated molecular pattern, MAMP)的作用激发植物的 防卫反应 ${ }^{[1]}$ 。拟南芥受体激酶 AtCERK1 (chitin elicitor receptor kinase 1 of Arabidopsis)的胞外区有 3 个赖氨酸基序(lysine motif, LysM), 它们可以与几丁 质臭糖结合从而激活植物免疫反应。几丁质诱发的 AtCERK1 的二聚化可进一步激发 AtCERK1 介导的 信号途径 ${ }^{[2]}$ 。在水稻中, 第一个报道的几丁质酶寡糖 受体是 CEBiP (chitin elicitor binding protein), 它带 有 2 个 LysM 的糖蛋白, 与几丁质寡糖具有很高的亲 和力, CEBiP 和激酶 OsCERK1 共同作用, 可使植物 对来自几丁质葟糖的信号做出反应 ${ }^{[3-4]}$ 。最近又有报 道表明, LYP4 (lysine motif-containing protein 4)和 LYP6 与 CEBiP 具有同源性, 它们对水稻识别几丁质 信号也发挥着作用 ${ }^{[2]}$ 。将 CEBiP 与水稻白叶枯病抗 性基因 $X A 21$ 或稻瘟病抗性基因 $P I-D 2$ 重组形成嵌合 基因, 可以增强水稻对稻瘟病菌的抗性 ${ }^{[5-6]}$ 。

几丁质酶(EC 3.2.1.14)的作用是将几丁质分解为 $\mathrm{N}-$ 乙酰葡萄糖胺, 在自然界中, 几丁质酶在细菌、真 菌、植物甚至动物中都存在。根据作用于几丁质的方 式不同，几丁质酶可分为内切和外切几丁质酶二大 类 ${ }^{[7]}$, 根据氨基酸序列的相似性和几丁质结合结构域 的有无可把几丁质酶分为 Class I Class VII ${ }^{[8-9]}$ 。

有大量报道表明，几丁质酶在植物中的表达受 生物或非生物胁迫的诱导。在拟南芥中, 非亲和病 原物的侵染使 Class IV 几丁质酶基因的转录更快地 积累 ${ }^{[10]}$, 而用亲和病原物侵染可使 Class III 几丁质 酶的转录升高 ${ }^{[11]}$ 。在甜菜中也观察到亲和病原物的 侵染可使 Class IV 几丁质酶转录增强的现象 ${ }^{[12]}$ 。从 稻瘟病菌侵染后的叶片 cDNA 中克隆出一个属于 Class III 几丁质酶编码基因, 命名为 $O s c h i b 1$, 该基 因的转录在稻瘟病菌和白叶枯病菌侵染后均大幅提 高, 且在接种非亲和稻瘟病菌的组织中 Oschibl 反 应更快速, 另外, 该基因的转录提升受水杨酸 (salicylic acid, SA)、乙烯、甲基茉莉酸、 $\mathrm{H}_{2} \mathrm{O}_{2}$ 和 $\mathrm{CuSO}_{4}$ 的诱导 ${ }^{[13]}$ 。

也有多例报道表明，几丁质酶在正常生长的植 物组织中也有表达。如在烟草的花形成阶段 ${ }^{[14]}$ 、在 葡萄的果实成熟期 ${ }^{[15]}$ 以及水稻的叶片、叶鞘、根和 分生组织中都有高表达的几丁质酶 ${ }^{[9]}$ 。胡夢卜的EP3
几丁质酶在体细胞胚胎发生过程中发挥作用，拟南 芥的ep3 同源基因为Atep $3 /$ Atchit $I V$, 该基因在胚胎 细胞的周围看护细胞中表达, 但不在胚胎细胞中表 达，在拟南芥植株中，该基因在成熟的花粉和生长 中的花粉管中表达，但没有检测到在胚乳和珠被中 的转录, 在胚胎形成后期, 其转录还发生在托叶、根 表皮和根毛中 ${ }^{[16]}$ 。拟南芥类几丁质酶基因 $A t c t l l$ 的突 变引起了木质素的异位沉积和细胞的非正常形态突 变, Atctll 基因在大部分正常生长的组织中表达, 但 不受创伤、SA或乙烯处理的诱导 ${ }^{[17]}$ 。水稻脆秆突变 体 $b c 15$ 的控制基因编码几丁质酶类蛋白 $\mathrm{BC} 15$ / OsCTL1, 该基因的突变引起厚壁细胞壁变薄和纤 维素含量下降，导致水稻茎秆机械强度降低，表达 模式检测发现BC15/OsCTL1在水稻的各个组织器官 中均广泛表达 ${ }^{[18]}$ 。这些事实说明几丁质酶不但在逆 境胁迫反应中有作用, 在植物正常生长发育过程中 也发挥着作用。

转基因实验也提供了几丁质酶作用的证据。在 水稻中组成型表达 Cht2 (Os05g33130) 或 Cht3 (Os06g51050)基因提高了对稻瘟病的抗性，且几丁 质酶的高水平表达和稻瘟病抗性在后代中能稳定遗 传 ${ }^{[19-20]}$ 。在水稻中组成型表达几丁质酶基因 $R C 7$, 使 水稻增强了抗纹枯病的能力 ${ }^{[19-20]}$ 。干扰编号为 Os02g39330 或 Os04g41620 的水稻几丁质酶基因的 表达, 会使水稻提高对稻瘟病菌及白叶枯病菌的敏感 性 ${ }^{[21]}$ 。把水稻的几丁质酶基因转入香蕉或小麦 ${ }^{[22-23]}$ 可 提高受体植物的抗病性。

白叶枯病是危害水稻最为严重的细菌性病害, 在水稻育种和植病学家的努力下，先后鉴定了 30 多 个水稻白叶枯病抗性基因，其中7个已被克隆 ${ }^{[24]}$ 。最 早克隆的 Xa21编码受体激酶, 且对白叶枯病菌具有 广谱抗性，过去十几年中，通过酵母双杂交和 Pull-down 技术已经鉴定了 $X B 3 、 X B 15 、 X B 24 、 B i p 3^{[25-28]}$ 等多个在 $X a 21$ 介导的抗病途径中的关键元件, 利用 水稻全基因组基因芯片体系，我们篮选出了441个 在 Xa21 介导的抗病反应中的差异转录基因。采用基 于抗体的蛋白质组学策略, 利用免疫印迹技术 (western blot, WB), 我们也检测到 PR、WRKY 转录 因子等抗病相关蛋白质的表达变化 ${ }^{[24,29-32]}$ 。

综上所述，几丁质酶在植物正常生长发育和抗 病过程中都可能发挥作用。水稻中的几丁质酶属于 PR3 家族, 有 19 个成员 ${ }^{[33]}$, 本研究以水稻 PR3 家族 的几丁质酶为对象, 分析了几丁质酶基因的转录和 
蛋白质表达特征, 试图为水稻几丁质酶的功能研究 提供线索。

\section{1 材料与方法}

\section{1 试验材料}

水稻品种 93-11 是超级杂交稻两优培九的父本, 4021 是将白叶枯病抗性基因 Xa21 转入水稻品 TP309后获得的纯合转基因株系，对白叶枯病菌 PXO99 野生型(由中国科学院遗传与发育生物学研 究所朱立煌研究员提供)表现不亲和反应, 对白叶枯 病菌突变株 PXO99 $\Delta$ raxST (由美国加州大学戴维斯 分校 Pamela Ronald 教授提供)表现亲和反应。

\section{2 试验方法}

1.2.1 水稻接种与取材 在 PSA 培养基中 $(0.5 \%$ bacto peptone, $2 \%$ sucrose, $0.05 \% \mathrm{~L}$-glutamic acid) $30^{\circ} \mathrm{C}$ 培养白叶枯病菌 48 72 h 后, 用水稀释至 $10^{9}$ 个细胞 $\mathrm{mL}^{-1}$, 用灭菌剪刀蘸取菌液剪切叶片以接种, 以灭 菌蒸馏水作为对照(Mock)。分别于接种后 $0 \mathrm{~h} 、 2 \mathrm{~h}$ 、 $8 \mathrm{~h} 、 1 \mathrm{~d} 、 2 \mathrm{~d} 、 3 \mathrm{~d} 、 5 \mathrm{~d} 、 7 \mathrm{~d}$ 和 $10 \mathrm{~d}$ 采集接种部位 叶片(约 $1 \mathrm{~cm}$ ), 液氮速冻后于 $-70^{\circ} \mathrm{C}$ 冰箱保存。

\subsection{2 多克隆抗体制备目标几丁质酶蛋白质序}

列来自水稻基因组数据库 (http://rice.plantbiology. msu.edu), 利用 BEPITOPE 软件预测抗原决定簇, 选 择峰值较高的片段, 用 BLASTP 软件对水稻蛋白质 库进行唯一性检测后, 确定目标多肽片段。以合成 多肽作为免疫原, 多克隆抗体的制备由北京华大蛋 白质研发中心有限公司完成。

1.2.3 水稻蛋白质提取 将 $-70^{\circ} \mathrm{C}$ 冻存的水稻叶 片在液氮中充分研磨, 加入蛋白质提取液 $(62.5$ mmol L ${ }^{-1}$ Tris- $\mathrm{HCl}, \mathrm{pH}$ 7.4；10\%甘油；0.1\% SDS; $2 \mathrm{mmol} \mathrm{L}{ }^{-1}$ EDTA; $1 \mathrm{mmol} \mathrm{L}^{-1} \mathrm{PMSF} ; 5 \% \beta$-颈基乙 醇), 混匀, 冰上放置 $10 \mathrm{~min}, 12000$ 转 $\mathrm{min}^{-1} 4^{\circ} \mathrm{C}$ 离 心 $20 \mathrm{~min}$, 取上清液即为总蛋白质, 具体方法可参 考本课题组以前的报道 ${ }^{[29]}$ 。

1.2.4 WB 分析和信号采集 WB 分析实验至少 重复 3 次, 使用 HSP (heat shock protein)蛋白质的检 测信号作为等量加样的内参 ${ }^{[34]}$ 。用 ImageJ 软件扫描 $\mathrm{X}$ 光片上的条带信号, 计算平均值及标准差, 比较 其信号的相对强度。

1.2.5 生物信息学分析 从水稻基因组数据库中, 根据几丁质酶蛋白质的 Locus 号, 获得几丁质酶基 因编码的蛋白质序列, 使用 ClustalW 进行多序列比 对, 参数使用默认值, 使用 Mega4.0 进行系统树分
析，应用临近法, boot strap 次数为 2000 。用 SMART 软件(http://smart.embl-heidelberg.de/)进行结构域分 析。从水稻基因组注释项目 (rice genome annotation project)数据库(http://rice.plantbiology.msu.edu/)找到 目标几丁质酶基因后下载 RNA-Seq 产生的 FPKM (reads per kilobase of exon model per million mapped reads)数据, 其高通量测序产生的转录原始数据可从 NCBI 的 SRA (Sequence Read Archive)数据库(http:// www.ncbi.nlm.nih.gov/sra)根据文库的编号下载。

\section{2 结果与分析}

\section{1 水稻 PR3 家族的几丁质酶基因}

水稻 PR3 家族中有 19 个几丁质酶成员 ${ }^{[33]}$, 表 1 列出了这些几丁质酶编码基因的 LOC 号、注释和分 子量, 水稻 PR3 家族几丁质酶蛋白质的分子量大都 在 $30 \mathrm{kD}$ 左右, 最高为 $36.7 \mathrm{kD}$, 最低的为 CHIT9, 只有 $15.3 \mathrm{kD}$ (154 个氨基酸)。用 SMART 软件预测 几丁质酶的结构域(图 1), 可以看出几丁质酶的主要 结构域有 $\mathrm{N}$ 端的信号肽、几丁质结合域和催化域, 其中, CHITC1 和 CHITC2 的催化域属于糖苷酶 18 家族，而其余几丁质酶的催化域都属于糖苷酶 19 家 族。需要指出的是, CHIT9 中没有明显的几丁质结合 域和催化域。Nakazaki 等 ${ }^{[9]}$ 曾将当时鉴定的 12 个水 稻 PR3 几丁质酶划分了类别(Class), 在此基础上, 我们补充了新鉴定的 7 个几丁质酶, 将 19 个水稻 PR3 几丁质酶进行了分类(图 2), 类别编号列于聚类图的 右侧, 其中最初鉴定的 12 个水稻 PR3 几丁质酶的分 类结果与原报道相同。从图 2 可见，大部分编号相 近的几丁质酶的相似度较高, 如 CHIT7 和 CHIT8, CHIT10 和 CHIT11, CHIT12 和 CHIT13, CHIT2 和 CHIT3 以及 CHITC1 和 CHITC2 等。其中 CHITC1 和 CHITC2 与其他几丁质酶的亲缘关系较远，但整 个 PR3 家族几丁质酶的相似度较高。属于 Class I 的 几丁质酶有 11 个成员, 缺失几丁质结合域的 Class II 成员有 CHIT14、CHIT15 和 CHIT16，拥有糖苷酶 18 家族的催化域的 Class III 成员有 CHITC1 和 CHITC2, 而 CHIT1、CHIT2 和 CHIT3 的催化域中有 4 段缺失, 它们组成了 Class VI 亚类。

\section{2 几丁质酶基因的转录分析}

经由 RNA-Seq 分析获得了多个水稻组织(幼苗 期地上部或叶片、穗部、花药及雌蕊、授粉后未成 熟胚和胚乳等)的转录的 FPKM 数据(表 2), 比较不 同几丁质酶基因在参试组织中的转录强度可见， 


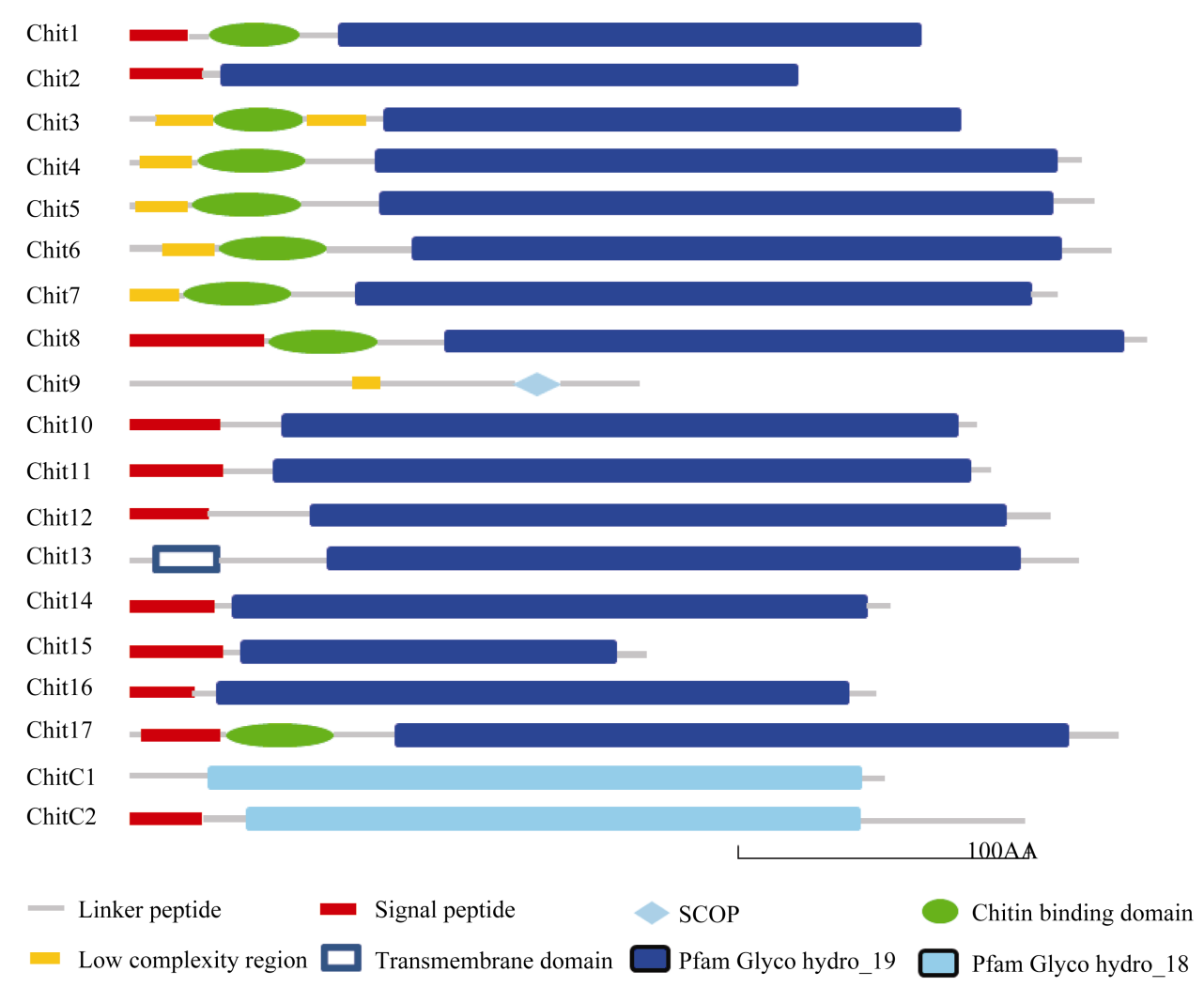

图 1 水稻 PR3 家族几丁质酶的结构域

Fig. 1 Predicted domains of rice PR3 family chitinases

表 1 水稻 PR3 家族几丁质酶编码基因的相关信息

Table 1 Information of rice PR3 family chitinase genes

\begin{tabular}{lllc}
\hline 基因 & 编号 & \multicolumn{1}{c}{$\begin{array}{c}\text { 简要注释 } \\
\text { Brief annotation }\end{array}$} & $\begin{array}{c}\text { 分子量 } \\
\text { Molecular weight (kD) }\end{array}$ \\
\hline Chit1 & Os02gs code & \multicolumn{1}{c}{ CHIT1-Chitinase family protein precursor, expressed } & 28.5 \\
Chit2 & Os04g41620 & CHIT2-Chitinase family protein precursor, expressed & 25.2 \\
Chit3 & Os04g41680 & CHIT3-Chitinase family protein precursor, expressed & 30.5 \\
Chit4 & Os03g30470 & CHIT4-Chitinase family protein precursor, expressed & 33.6 \\
Chit5 & Os05g33140 & CHIT5-Chitinase family protein precursor, expressed & 34.4 \\
Chit6 & Os05g33150 & CHIT6-Chitinase family protein precursor, expressed & 35.3 \\
Chit7 & Os06g51050 & CHIT7-Chitinase family protein precursor, expressed & 33.8 \\
Chit8 & Os06g51060 & CHIT8-Chitinase family protein precursor, expressed & 36.7 \\
Chit9 & Os12g13610 & CHIT9-Chitinase family protein precursor, expressed & 15.3 \\
Chit10 & Os01g18400 & CHIT10-Chitinase family protein precursor, expressed & 31.3 \\
Chit11 & Os05g04690 & CHIT11-Chitinase family protein precursor, expressed & 32.1 \\
Chit12 & Os08g41100 & CHIT12-Chitinase family protein precursor, expressed & 34.6 \\
Chit13 & Os09g32080 & CHIT13-Chitinase family protein precursor, expressed & 36.5 \\
Chit14 & Os10g39680 & CHIT14-Chitinase family protein precursor, expressed & 27.5 \\
Chit15 & Os10g39700 & CHIT15-Chitinase family protein precursor, expressed & 18.9 \\
Chit16 & Os03g04060 & CHIT16-Chitinase family protein precursor, expressed & 27.7 \\
Chit17 & Os05g33130 & CHIT17-Chitinase family protein precursor, expressed & 35.6 \\
ChitC1 & Os01g43220 & Chitinase domain-containing protein 1 precursor, putative & 29.7 \\
ChitC2 & Os10g28050 & Chitinase 2-putative, expressed & 33.7 \\
\hline
\end{tabular}




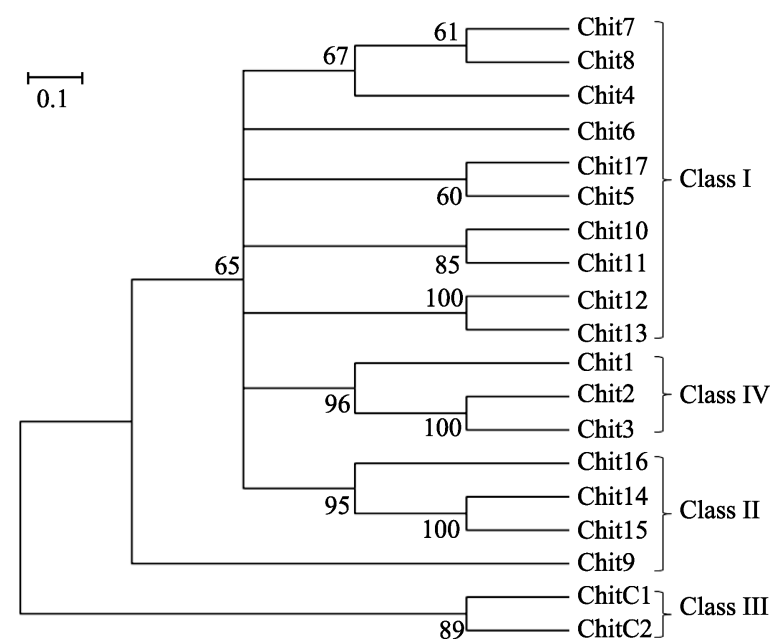

图 2 基于氨基酸序列同源性的水稻几丁质酶蛋白质聚类分析 Fig. 2 Cluster analysis of rice chitinase proteins based on amino acid sequences

用 Mega4.0 进行系统树分析, 应用临近法构建, 并做 2000 次检验。 分支上的数字为贝叶斯后验概率, 分支长度表示每个核苷酸的替 代率, 对应每个位点 0.1 个估计的替换的标尺列于图的左上方。 Tree: Mega4.0, Neighbor-Joining, bootstrap=2000; Bayesian posterior probabilities are showed by number of branch; the length of branch represents the substitution rate of each nucleotide, estimated replacement corresponding scale shown in the upper left graph.

Chit6 的 FPKM 值最高, 表明其转录强度高, 其转录 主要发生在穗部, 尤其是在雌芯芯中, 而在其他组织 中的转录强度较低, 该基因的转录呈现明显的组织 特异性。Chit12、Chit13、ChitC2 和 Chit5 在大部分
参试组织中都有一定的转录信号, 呈现组成型转录 的特点。Chit9 在所有参试材料中都没有被检测到转 录信号, 结合 CHIT9 的结构特征可以推测该基因具 有某种特殊性。整体来看, 除 Chit6 在穗部表达量较 高外，大部分 Chit 基因在水稻苗期叶片和授粉后的 种子或胚乳中表达量均较低。根据 RNA-Seq 提供的 转录信息对几丁质酶也进行了聚类分析，结果见图 3 。比较图 2 和图 3 结果可见, 2 种方法聚类的结果 有相似之处, 说明序列相似、进化关系较近的基因 可能有相似的转录谱, 表达谱的相似性支持其功能 上的互补性。

2.3 几丁质酶蛋白质在水稻叶片生长过程中的 表达分析

为了解水稻 PR3 家族几丁质酶蛋白质的表达特 征，我们制备了几丁质酶蛋白质特异的抗体，采用 免疫印迹技术分析了不同时期的水稻叶片，检测到 部分几丁质酶蛋白质在正常生长的水稻叶片中有表 达(图 4)。从图 4 可见, CHIT5 在幼苗期的表达强度 较高, 在 $15 \mathrm{~cm}$ 幼苗及后续时期样品中均检测不到 信号。CHIT6 和 CHIT11 表现为随叶片生长表达强 度逐步提高, 在开花期达到最高峰, 成熟期有所下 降, CHIT14 的表达上调在苗期即达到最高峰, 在以 后的时期保持恒定, CHITC2 的表达呈现逐步上升的 特点, 到成熟期达到最高峰。

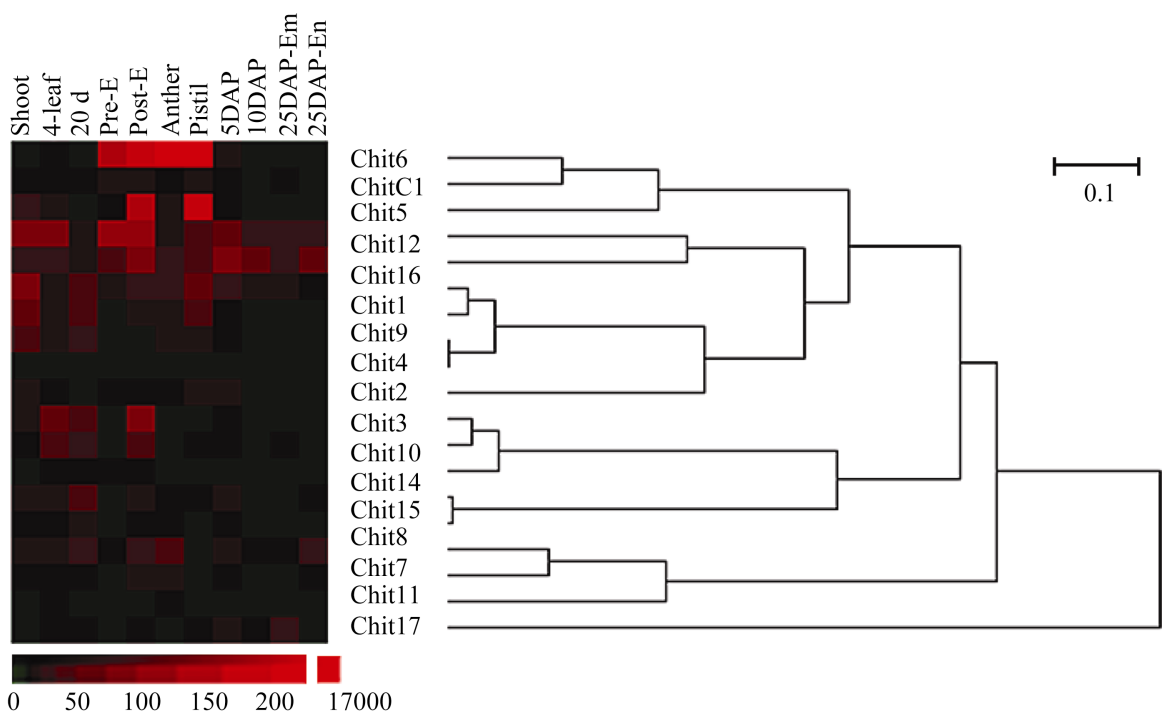

图 3 基于转录谱的水稻 PR3 家族几丁质酶的聚类分析

Fig. 3 Cluster analysis of rice chitinase genes in PR3 family based on transcription data

Shoot: 苗期地上部; 4-leaf: 四叶期; 20 d: 20 d 叶片; Pre-E: 花序出现前; Post-E: 花序出现后; Anther: 花药; Pistil: 雌荵;

DAP: 授粉后天数; Em: 胚; En: 胚乳。

Pre-E: pre-emergence inflorescence; Post-E: pre-emergence inflorescence; DAP: days after pollination; Em: embryo; En: endosperm. 
表 2 水稻几丁质酶基因在不同组织中的 FPKM 数据

Table 2 FPKM value of rice chitinases genes at different tissue/organs

\begin{tabular}{|c|c|c|c|c|c|c|c|c|c|c|c|c|}
\hline \multirow{2}{*}{$\begin{array}{l}\text { 基因 } \\
\text { Gene }\end{array}$} & \multicolumn{3}{|c|}{ 叶片 Leaf } & \multicolumn{2}{|c|}{ 花序 Inflorescence } & \multirow{2}{*}{$\begin{array}{c}\text { 花药 } \\
\text { Anther }\end{array}$} & \multirow{2}{*}{$\begin{array}{l}\text { 雌芯芯 } \\
\text { Pistil }\end{array}$} & \multicolumn{4}{|c|}{ 种子 Seed } & \multirow{2}{*}{$\begin{array}{l}\text { 合计 } \\
\text { Total }\end{array}$} \\
\hline & $\begin{array}{c}\text { 地上部 } \\
\text { Shoot }\end{array}$ & $\begin{array}{c}\text { 四叶期 } \\
\text { 4-leaf stage }\end{array}$ & $20 \mathrm{~d}$ & Pre-E & Post-E & & & 5 DAP & $10 \mathrm{DAP}$ & $\begin{array}{c}25 \\
\text { DAP-Em }\end{array}$ & $\begin{array}{c}25 \\
\text { DAP-En }\end{array}$ & \\
\hline Chit1 & 27.5 & 6.9 & 13.9 & 0 & 0 & 4.7 & 3.7 & 1.7 & 0 & 0 & 0 & 58.4 \\
\hline Chit2 & 5.1 & 42.2 & 29.6 & 0 & 70.3 & 0 & 0 & 1.0 & 0 & 0 & 0 & 148.1 \\
\hline Chit 3 & 1.8 & 27.9 & 16.2 & 0 & 27.8 & 0 & 0.5 & 1.2 & 0 & 0.6 & 0 & 75.9 \\
\hline Chit4 & 3.9 & 2.4 & 0 & 2.2 & 0.6 & 0.6 & 6.6 & 6.1 & 0 & 0 & 0 & 22.5 \\
\hline Chit5 & 12.6 & 3.0 & 0 & 2.5 & 137.1 & 7.2 & 174.2 & 0.7 & 0 & 0 & 0 & 337.4 \\
\hline Chit6 & 0 & 1.0 & 0 & 203.2 & 7592.8 & 857.8 & 16011.2 & 7.5 & 0 & 0 & 0 & 24673.6 \\
\hline Chit7 & 0.7 & 1.5 & 2.5 & 0 & 2.6 & 9.1 & 0 & 2.1 & 0 & 0 & 1.7 & 20.1 \\
\hline Chits & 4.7 & 6.3 & 22.0 & 0.6 & 15.2 & 25.9 & 0 & 5.1 & 1.7 & 1.4 & 16.6 & 99.4 \\
\hline Chit 9 & 0 & 0 & 0 & 0 & 0 & 0 & 0 & 0 & 0 & 0 & 0 & 0 \\
\hline Chit10 & 0 & 2.2 & 1.8 & 0.5 & 1.8 & 0 & 0 & 0 & 0 & 0 & 0 & 6.3 \\
\hline Chit11 & 0 & 0.4 & 0 & 0 & 0 & 1.0 & 0 & 0 & 0 & 0 & 0 & 1.3 \\
\hline Chit12 & 21.3 & 22.4 & 7.1 & 23.9 & 69.9 & 16.3 & 34.6 & 77.7 & 60.6 & 18.9 & 54.8 & 407.5 \\
\hline Chit13 & 79.0 & 70.0 & 7.5 & 91.7 & 99.9 & 6.7 & 24.7 & 44.7 & 15.5 & 10.2 & 21.0 & 470.9 \\
\hline Chit14 & 4.4 & 5.0 & 27.0 & 0 & 2.6 & 1.9 & 0.7 & 4.1 & 0 & 0 & 0.5 & 46.3 \\
\hline Chit15 & 1.3 & 0.8 & 5.1 & 0 & 0.5 & 0.3 & 0 & 0.9 & 0 & 0 & 0 & 9.0 \\
\hline Chit16 & 40.1 & 3.5 & 22.8 & 0 & 9.6 & 4.5 & 26.9 & 2.5 & 0 & 0 & 0 & 109.9 \\
\hline Chit17 & 0 & 0.9 & 0.8 & 0 & 1.3 & 0 & 1.0 & 4.4 & 0.5 & 13.5 & 0 & 22.4 \\
\hline ChitCl & 0.3 & 0.8 & 0.7 & 6.1 & 5.1 & 1.8 & 5.1 & 2.1 & 0 & 1.7 & 0 & 23.7 \\
\hline ChitC2 & 79.1 & 5.4 & 27.8 & 5.5 & 16.6 & 17.0 & 44.9 & 12.3 & 2.7 & 3.2 & 2.4 & 216.8 \\
\hline 小计 Sub-total & 281.7 & 202.7 & 184.7 & 336.3 & 8053.8 & 954.7 & 16334.2 & 174.1 & 81.1 & 49.5 & 96.9 & 26749.8 \\
\hline
\end{tabular}

FPKM：每百万被测到的外元中每一千碱基所含目标片段数; Pre-E：花序出现前; Post-E：花序出现后; DAP：授粉后天数; Em：胚; En: 胚乳。表中数据来源的文库编号: 苗期地上部叶片: SRR042529; 四叶期: SRX016110; $20 \mathrm{~d}$ 叶片: OSN_AA; 花序出现前: OSN_AC; Post-E: 花序出现后: OSN_AB; 花药: OSN_AD; 雌荵: OSN_AE; 授粉后 5 d: OSN_AF; 授粉后 10 d: OSN_AK; 胚: OSN_AG; 胚乳: OSN_AH。

FPKM: fragments per kilobase of exon per million fragments mapped; Pre-E: pre-emergence inflorescence; Post-E: post-emergence inflorescence; DAP: Days after pollination; Em: embryo; En: endosperm. Data derived from Libraries: Shoots: SRR042529;4-leaf: SRX016110;20 d: OSN_AA; Pre-E: OSN_AC; Post-E: OSN_AB; Anther: OSN_AD;Pistil:OSN_AE; 5DAP: OSN_AF; 10DAP: OSN_AK; Em: OSN_AG; En: OSN_AH.
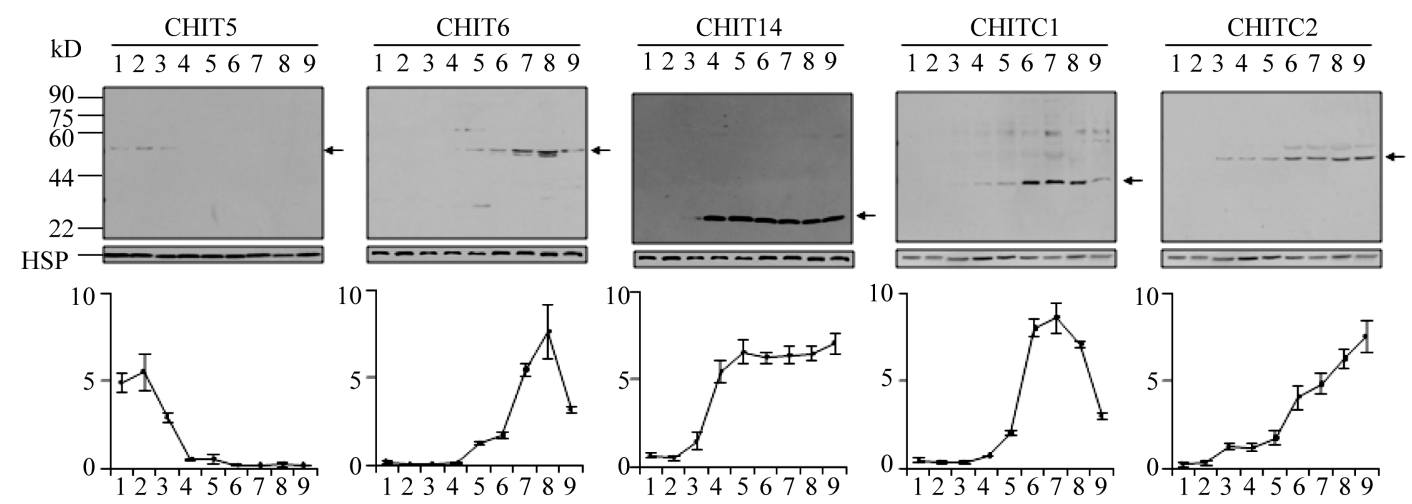

图 4 水稻 PR3 家族几丁质酶蛋白质在叶片中的表达谱

Fig. 4 Expression patterns of rice PR3 family chitinase proteins in normal growth leaves

上部图片为相应 CHIT 特异抗体检测水稻叶片蛋白质的 WB 结果; 中部为用 HSP 抗体检测的 WB 结果(示样品的等量加样); 下部为采 集 3 次 WB 数据, 计算平均值和方差绘制的折线图, 纵坐标为相对信号强度, 横坐标与泳道对应。泳道 1 9 分别代表水稻的苗期 $1 \mathrm{~cm}$ 、

$2 \mathrm{~cm} 、 5 \mathrm{~cm} 、 10 \mathrm{~cm}$ 和 $15 \mathrm{~cm}$ 地上部，分菜期叶片、孕穗期叶片、开花期叶片和成熟期叶片的总蛋白质样品。

Upper panels: WB detection of the expression of CHIT at different time points in rice leaves; Middle panels: WB detection of HSP expression in rice leaves to demonstrate equal loading; Lower panels: Lanes 1-9: Protein samples isolated from normal rice leaves at seedling stage

$(1 \mathrm{~cm}, 2 \mathrm{~cm}, 5 \mathrm{~cm}, 10 \mathrm{~cm}$, and $15 \mathrm{~cm})$, tillering stage, booting stage, flowering stage and mature stage, respectively. 
2.4 几丁质酶蛋白质在水稻抗白叶枯病过程中 的表达

白叶枯病是危害水稻生产最为严重的细菌性病害, 为了检测 CHIT 蛋白质在水稻抗病过程中的表达, 我 们将白叶枯病菌接种到带有白叶枯病抗性基因 Xa21 的转基因水稻 4021 叶片上。为了确证 4021 对白叶枯 病菌的抗性反应, 我们在叶片接种后 $15 \mathrm{~d}$, 量取叶片 病斑长度, 发现 4021 的病斑长度在 $1 \mathrm{~cm}$ 以下, 呈现典 型的抗病反应, 而接种 TP309 后的病斑长度在 $18 \mathrm{~cm}$ 以上, 呈现典型的感病特征, 同时接种蒸馏水的 Mock
对照没有明显的病玟，水稻接种白叶枯病菌后的表型 变化与我们以前的报道一致 ${ }^{[24]}$ 。同时，在接种后不同 时间点取样, 提取总蛋白质进行 WB 分析(图 5)。由图 5 可见，随接种后时间的延长, CHIT1、CHIT2、CHIT5、 CHIT6、CHIT10、CHIT15 和 CHIT16 蛋白质都上调或 诱导表达, 而 CHIT14、CHITC1 和 CHITC2 均下调表 达。由图 5 还可看出, 可检测的蛋白质表达变化大都 在接种后 $3 \mathrm{~d}$ 或以上。二个属于 Class III 的几丁质酶 蛋白质(CHITC1 和 CHITC2)都下调表达, 而其他 Class 的几丁质酶蛋白质大都上调表达。

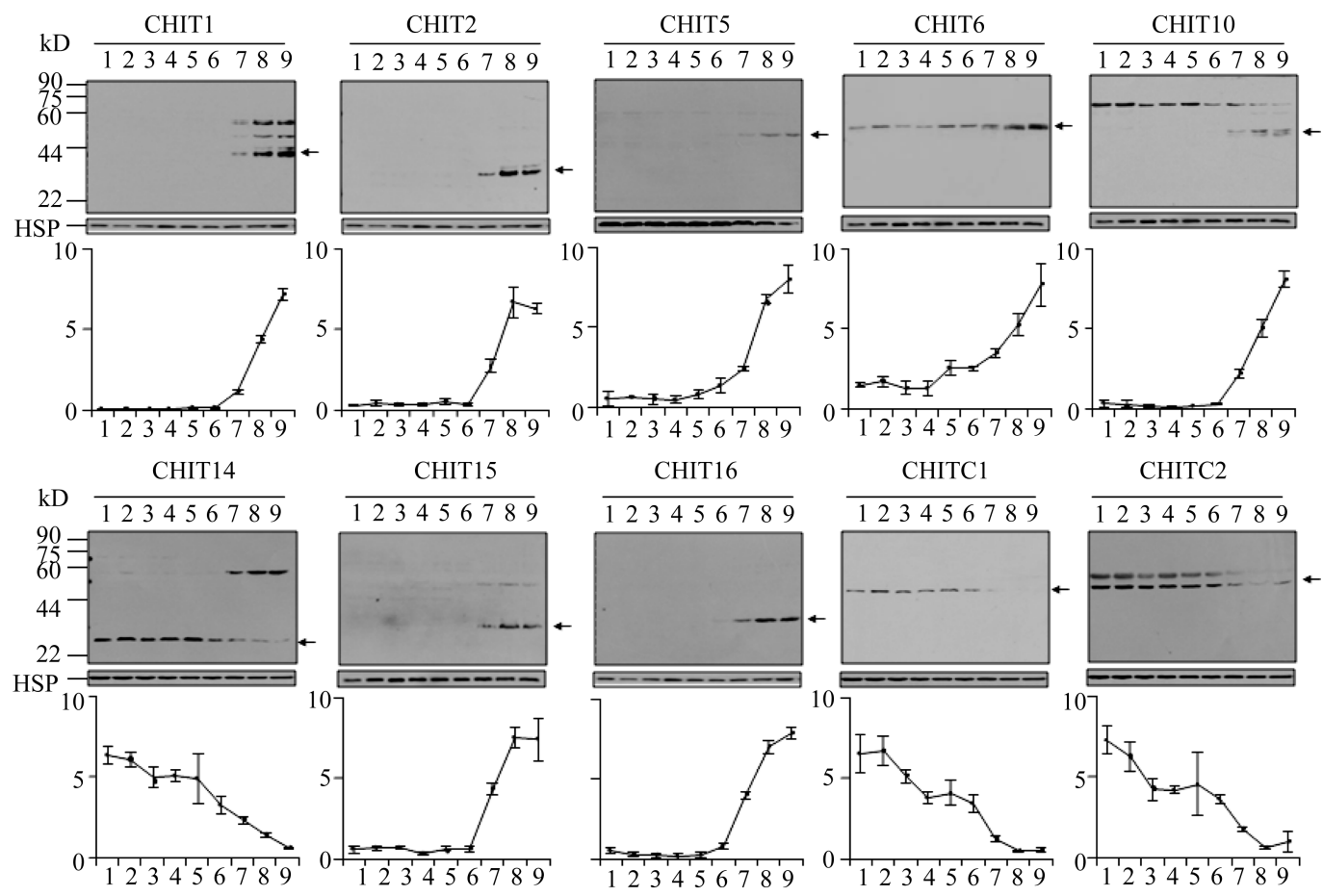

图 5 水稻PR3 几丁质酶蛋白质在抗白叶枯病反应中的表达谱

Fig. 5 Expression patterns of rice PR3 family chitinase proteins in the incompatible reactions between rice and Xoo 上部图片为相应 CHIT 特异抗体检测接种白叶枯病菌后不同时间点的水稻叶片蛋白质的 WB 结果; 中部为用 HSP 抗体检测的 WB 结 果(示样品的等量加样); 下部为采集 3 次 WB 数据, 计算平均值和方差绘制的折线图, 纵坐标为相对信号强度, 横坐标与泳道对应。泳 道 1 9 分别为接种后 $0 \mathrm{~h} 、 2 \mathrm{~h} 、 8 \mathrm{~h}$ 和 $1 \mathrm{~d} 、 2 \mathrm{~d} 、 3 \mathrm{~d} 、 5 \mathrm{~d} 、 7 \mathrm{~d} 、 10 \mathrm{~d}$ 的蛋白质样品。

Upper panels: WB detection of the expression of CHIT at different time points in the incompatible interaction between rice and Xoo; Middle panels: WB detection of HSP expression in rice leaves to demonstrate equal loading; Lower panels: plot of average and standard deviation among three repeats of WB analysis. Lanes 1-9: protein samples isolated from rice leaves at $0 \mathrm{~h}, 2 \mathrm{~h}, 8 \mathrm{~h}, 1 \mathrm{~d}, 2 \mathrm{~d}, 3 \mathrm{~d}, 5 \mathrm{~d}, 7 \mathrm{~d}$, and $10 \mathrm{~d}$ after inoculation with Xoo, respectively.

2.5 几丁质酶蛋白质在不同水稻-Xoo 互作反应 中的表达比较

比较几丁质酶蛋白质在 3 个时间点 $(0 \mathrm{~h} 、 3 \mathrm{~d}$ 和 5 d)在抗病(R: 4021-PXO99)、感病(S: 4021-PXO99 $\Delta \mathrm{raxST}$ )和对照 $\left(\mathrm{M}: 4021-\mathrm{H}_{2} \mathrm{O}\right.$ )反应中的表达表明(图 6), 在抗病和感病反应中, 各几丁质酶蛋白质的表
达具有相近的模式，与 Mock 反应都有明显的区别, 且一般在感病反应中的变化程度高于抗病反应。有 意思的是, CHIT6 在 Mock 反应中的表达量表现上升, 说明其表达可能受创伤的诱导, 其他几丁质酶蛋白 质表达量在 Mock 反应中表现一致, 说明这些蛋白 质的表达不受创伤的诱导。 


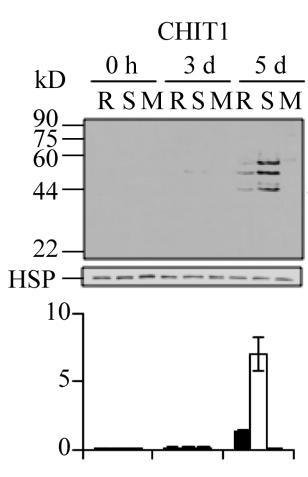

CHIT14

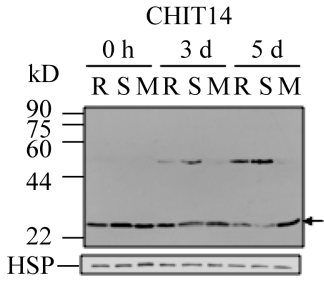

CHIT2
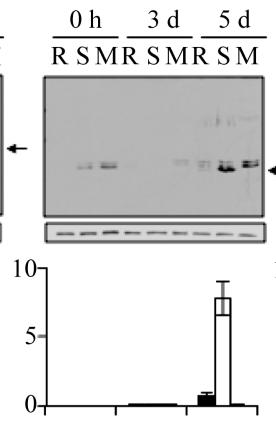

CHIT15
CHIT5
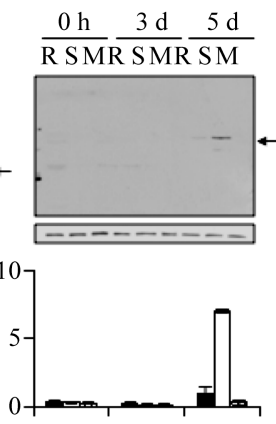

CHIT16
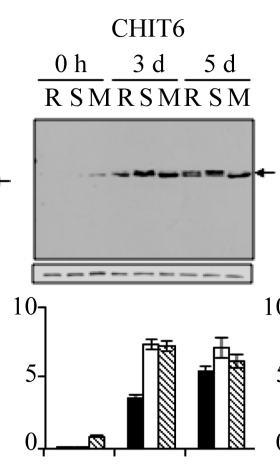

CHITC1
CHIT10
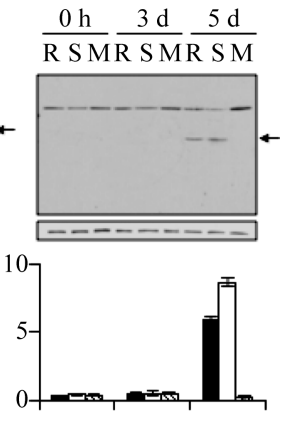

CHITC2

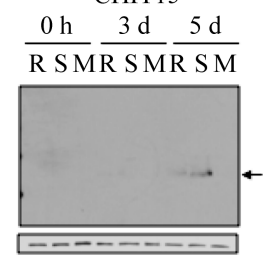

$\frac{0 \mathrm{~h}}{\text { R SMR SMR SM }}$

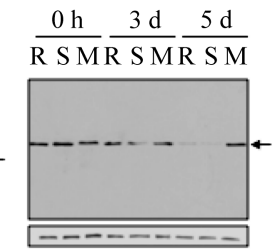

$\frac{0 \mathrm{~h}}{\operatorname{R~SM}} \frac{3 \mathrm{~d}}{\operatorname{R~SM}} \frac{5 \mathrm{~d}}{\mathrm{R} \mathrm{S} \mathrm{M}}$
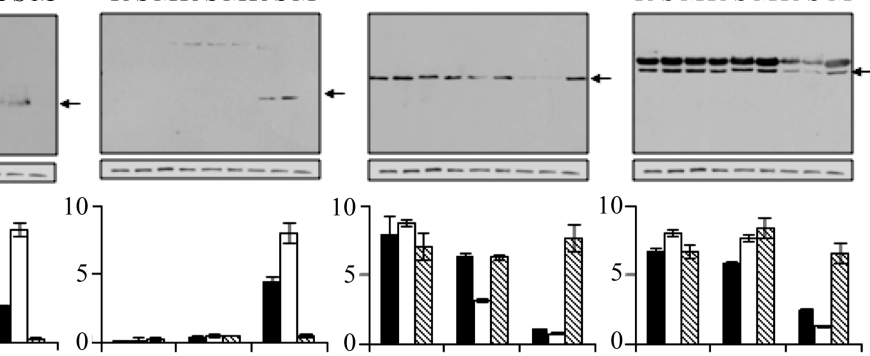

10

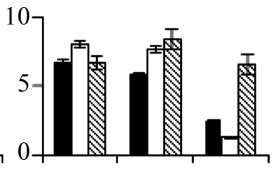

图 6 PR3 家族几丁质酶蛋白质在不同水稻-Xoo 互作反应中的表达比较

Fig. 6 Expression comparison of rice PR3 family chitinases proteins among different rice-Xoo interactions $0 \mathrm{~h} 、 3 \mathrm{~d}$ 和 $5 \mathrm{~d}$ 为水稻接种白叶枯病菌后的时间点; R: 抗病反应; $\mathrm{S}$ : 感病反应; M: Mock 对照。上部图片为用相应 CHIT 特异性抗体检 测的 WB 结果; 中部为用 HSP 抗体检测的 WB 结果(示样品的等量加样); 下部为采集 3 次 WB 数据, 计算平均值和方差绘制的框图, 纵 坐标为相对信号强度, 横坐标与泳道对应(实心柱、空心柱和线条柱分别表示 $R 、 S$ 和 M 的数据)。

$0 \mathrm{~h}, 3 \mathrm{~d}$, and $5 \mathrm{~d}$ were the time points post inoculation with Xoo; R: incompatible interaction; S: compatible interaction; M: mock control. Upper panels: WB detection of the expression of CHIT; Middle panels: WB detection of HSP expression in rice leaves to demonstrate equal loading;

Lower panels: plot of average and standard deviation among three repeats of WB analysis (black bars, white bars and lined bars designate the data of $\mathrm{R}, \mathrm{S}$, and $\mathrm{M}$, respectively).

\section{3 讨论}

有大量报道表明, 植物几丁质酶在抵抗生物或 非生物逆境胁迫及生长发育过程中发挥作用。近年 来, 经由几丁质与受体激酶的结合启动抗病信号传 导途径的发现进一步激发了人们对几丁质酶研究的 兴趣。水稻基因组序列的解析为在全基因组水平系 统开展几丁质酶家族的研究提供了可能。

在完成水稻全基因组序列解析和基因预测及注 释的基础上, 人们可以对不同基因家族的整体情况 有更为全面的了解。通过系统的分析发现, 水稻的 病程相关基因(PR)有900多个, 占全基因组编码基因 的 $2 \%$ 左右, 其中 PR3 为几丁质酶, 在水稻中注释为 几丁质酶的基因共有 19 个 $^{[33]}$ 。根据基因组注释提供 的几丁质酶氨基酸序列, 我们对几丁质酶进行了聚 类分析, 进而对其蛋白质结构域进行了预测, 并将 其划分为 4 个亚类。随着高通量测序技术的进步, 基 于深度测序技术获得的转录谱数据更为灵敏、准确, 为转录谱的分析提供了重要的资源。本试验发现,
在正常生长的水稻组织中, 几丁质酶基因的转录是 相当普遍的, 其转录也佐证了这些基因在正常发育 过程中可能发挥功能。Chit6 在雌惢中有很高的转录 丰度, 是典型的组织特异性转录基因, 还发现了多 个组成型转录的几丁质酶基因, 也注意到在所有参 试材料中未检测到 Chit 9 表达。

基于氨基酸序列相似性和基于表达谱相似性的 二种聚类分析表明, 二者间有一定的相似性, 前者 可能来自进化过程中的关联，后者可能支持其功能 的互补性, 暗示亲缘关系相近的基因更可能有功能 的互补性。随着转录谱数据的积累, 可能在更大范 围上分析基因转录的关联, 从而获得其功能以及功 能互补相关的线索。我们对 PR3 家族几丁质酶基因 的分析可以说是进行了一个尝试。

检测水稻 PR3家族几丁质酶蛋白质在正常生长 及与白叶枯病菌互作的叶片中的蛋白质表达, 仅发 现 5 个几丁质酶蛋白质在正常叶片中有表达, 而在水 稻与 $X O O$ 不亲和反应中, 有 10 个几丁质酶蛋白质的 
表达发生了变化, 其中 CHIT1、CHIT2、CHIT5、 CHIT10、CHIT15和 CHIT16的表达都是受诱导后发 生的, 多个几丁质酶的表达受白叶枯病菌接种的诱 导, 提示它们在抗病反应中可能发挥作用。有 3 个几 丁质酶蛋白质的表达在接种白叶枯病菌后下调, 它 们是 CHIT14、CHITC1和 CHITC2, 提示它们可能发 挥负调控的作用, 另外, CHITC1和 CHITC2 都属于 ClassIII，提示其表达下调可能是该类别几丁质酶的 特征。有意思的是, CHIT6在对照(Mock)反应中表达 上调, 可能是受创伤诱导的基因, 暗示其启动子与 创伤的关联性。

我们试图分析转录和蛋白质表达数据的相关性, 但并没有发现二者的明显关联，实际上，转录和翻 译是二个层面的调控机制, 有大量的数据揭示了二 者的独立性。从转录和蛋白质表达特征都可以看出, 水稻几丁质酶在正常生长发育中可能发挥作用, 在 水稻-Xoo 互作过程中可能发挥作用。但值得指出的 是，在水稻中并没有发现几丁质，几丁质也不是白 叶枯病菌这类细菌的组成成分, 那么在水稻正常生 长中几丁质酶的底物是什么? 其功能又是通过什么 方式发挥的呢? 已经鉴定的水稻 $b c 15$ 基因编码几丁 质酶(Os09g32080, 本文中 Chit 13), 该基因也呈组成 型表达，但体外表达的蛋白质没有检测到几丁质酶 活性 ${ }^{[18]}$, 深入开展酶学分析将有助于了解其功能的 生化机理。

本文制备了水稻 PR3 家族几丁质酶蛋白质的抗 体，并用免疫印迹揭示了其在不同条件下的蛋白质 表达谱。这种基于抗体的策略 ${ }^{[35]}$ 可以对特定目标蛋白 质开展研究, 其灵敏度较高。此外, 这些抗体也将是 几丁质酶蛋白质定位、免疫沉淀等研究的重要资源。

本研究对水稻 PR3 家族的 19 个成员的聚类分析、 结构域分析和亚类划分, 并根据深度测序获得的转录 数据鉴定了组成型和组织特异型转录的基因, 在此基 础上调查了正常生长和收白叶枯病菌侵染后的水稻叶 片中的蛋白质表达特征, 发现随水稻的生长发育 CHIT6、CHIT14、CHITC1、CHITC2 为上调表达, CHIT5 为下调表达; 在水稻与 Xoo 互作中 CHIT1、CHIT2、 CHIT5、CHIT6、CHIT10、CHIT15 和 CHIT16 蛋白质 为上调表达, CHIT14、CHITC1 和 CHITC2 为下调表达, 另外, CHIT6 的表达可能受创伤诱导。

\section{References}

[1] Boller T. Chemoperception of microbial signals in plant cells. Annu Rev Plant Biol, 1995, 46: 189-214
[2] Liu T, Liu Z, Song C, Hu Y, Han Z, She J, Fan F, Wang J, Jin C, Chang J, Zhou J M, Chai J. Chitin-induced dimerization activates a plant immune receptor. Science, 2012, 336: 1160-1164

[3] Kaku H, Nishizawa Y, Ishii-Minami N, Akimoto-Tomiyama C, Dohmae N, Takio K, Minami E, Shibuya N. Plant cells recognize chitin fragments for defense signaling through a plasma membrane receptor. Proc Natl Acad Sci USA, 2006, 103: 11086-11091

[4] Shimizu T, Nakano T, Takamizawa D, Desaki Y, Ishii-Minami N, Nishizawa Y, Minami E, Okada K, Yamane H, Kaku H, Shibuya N. Two LysM receptor molecules, CEBiP and OsCERK1, cooperatively regulate chitin elicitor signaling in rice. Plant $J, 2010$, 64: 204-214

[5] Kishimoto K, Kouzai Y, Kaku H, Shibuya N, Minami E, Nishizawa Y. Perception of the chitin oligosaccharides contributes to disease resistance to blast fungus Magnaporthe oryzae in rice. Plant $J, 2010,64$ : 343-354

[6] Kouzai Y, Kaku H, Shibuya N, Minami E, Nishizawa Y. Expression of the chimeric receptor between the chitin elicitor receptor $\mathrm{CEBiP}$ and the receptor-like protein kinase Pi-d2 leads to enhanced responses to the chitin elicitor and disease resistance against Magnaporthe oryzae in rice. Plant Mol Biol, 2013, 81: 287-295

[7] Kumar S, Sharma R, Tewari R. Production of N-acetylglucosamine using recombinant chitinolytic enzymes. Indian $J$ Microbiol, 2011, 51: 319-325

[8] Kitajima S, Sato F. Plant pathogenesis-related proteins: molecular mechanisms of gene expression and protein function. $J$ Biochem, 1999, 125: $1-8$

[9] Nakazaki T, Tsukiyama T, Okumoto Y, Kageyama D, Naito K, Inouye $\mathrm{K}$, Tanisaka $\mathrm{T}$. Distribution, structure, organ-specific expression, and phylogenic analysis of the pathogenesis-related protein-3 chitinase gene family in rice (Oryza sativa L.). Genome, 2006, 49: 619-630

[10] de A Gerhardt L B, Sachetto-Martins G, Contarini M G, Sandroni M, de P Ferreira R, de Lima V M, Cordeiro M C, de Oliveira D E, Margis-Pinheiro M. Arabidopsis thaliana class IV chitinase is early induced during the interaction with Xanthomonas campestris. FEBS Lett, 1997, 419: 69-75

[11] Samac D A, Shah D M. Developmental and pathogen-induced activation of the Arabidopsis acidic chitinase promoter. Plant Cell, 1991, 3: 1063-1072

[12] Nielsen K K, Bojsen K, Roepstorff P, Mikkelsen J D. A hydroxyproline-containing class IV chitinase of sugar beet is glycosylated with xylose. Plant Mol Biol, 1994, 25: 241-257

[13] Park C H, Kim S, Park J Y, Ahn I P, Jwa N S, Im K H, Lee Y H. Molecular characterization of a pathogenesis-related protein 8 gene encoding a class III chitinase in rice. Mol Cells, 2004, 17: $144-150$

[14] Neale A D, Wahleithner J A, Lund M, Bonnett H T, Kelly A, Meeks-Wagner D R, Peacock W J, Dennis E S. Chitinase, beta-1,3-glucanase, osmotin, and extensin are expressed in tobacco explants during flower formation. Plant Cell, 1990, 2: 673-684

[15] Robinson S P, Jacobs A K, Dry I B. A class IV chitinase is highly expressed in grape berries during ripening. Plant Physiol, 1997, 114: $771-778$ 
[16] Passarinho P A, Van Hengel A J, Fransz P F, de Vries S C. Expression pattern of the Arabidopsis thaliana AtEP3/AtchitIV endochitinase gene. Planta, 2001, 212: 556-567

[17] Zhong R, Kays S J, Schroeder B P, Ye Z H. Mutation of a chitinase-like gene causes ectopic deposition of lignin, aberrant cell shapes, and overproduction of ethylene. Plant Cell, 2002, 14: 165-179

[18] Wu B, Zhang B, Dai Y, Zhang L, Shang-Guan K, Peng Y, Zhou Y, Zhu Z. Brittle culm15 encodes a membrane-associated chitinase-like protein required for cellulose biosynthesis in rice. Plant Physiol, 2012, 159: 1440-1452

[19] Datta K, Tu J, Oliva N, Ona I I, Velazhahan R, Mew T W, Muthukrishnan S, Datta S K. Enhanced resistance to sheath blight by constitutive expression of infection-related rice chitinase in transgenic elite indica rice cultivars. Plant Sci, 2001, 160: 405-414

[20] Nishizawa Y, Nishio Z, Nakazono K, Soma M, Nakajima E, Ugaki M, Hibi T. Enhanced resistance to blast (Magnaporthe grisea) in transgenic japonica rice by constitutive expression of rice chitinase. Theor Appl Genet, 1999, 99: 383-390

[21] Snelling J. Role of Oryza sativa Chitinases in Disease Resistance. MS Thesis of Colorado State University, 2005

[22] Kovacs G, Sagi L, Jacon G, Arinaitwe G, Busogoro J P, Thiry E, Strosse H, Swennen R, Remy S. Expression of a rice chitinase gene in transgenic banana ('Gros Michel', AAA genome group) confers resistance to black leaf streak disease. Transgenic Res, 2013, 22: 117-130

[23] Huang X, Wang J, Du Z, Zhang C, Li L, Xu Z. Enhanced resistance to stripe rust disease in transgenic wheat expressing the rice chitinase gene RC24. Transgenic Res, 2013, 22: 939-947

[24] Bai H, Lan J P, Gan Q, Wang X Y, Hou M M, Cao Y H, Li L Y, Liu L J, Hao Y J, Yin C C, Wu L, Zhu L H, Liu G Z. Identification and expression analysis of components involved in rice Xa21-mediated disease resistance signalling. Plant Biol (Stuttg), 2012, 14: 914-922

[25] Wang Y, Pi L, Chen X, Chakrabarty P K, Jiang J, De Leon A L, Liu G-Z, Li L, Benny U, Oard J. Rice XA21 binding protein 3 is a ubiquitin ligase required for full Xa21-mediated disease resistance. Plant Cell Online, 2006, 18: 3635-3646

[26] Park C J, Peng Y, Chen X, Dardick C, Ruan D, Bart R, Canlas P E, Ronald P C. Rice XB15, a protein phosphatase 2C, negatively regulates cell death and XA21-mediated innate immunity. PLoS Biol, 2008, 6(9): e231

[27] Chen X, Chern M, Canlas P E, Ruan D, Jiang C, Ronald P C. An
ATPase promotes autophosphorylation of the pattern recognition receptor XA21 and inhibits XA21-mediated immunity. Sci Signal, 2010, 107: 8029

[28] Park C J, Bart R, Chern M, Canlas P E, Bai W, Ronald P C. Overexpression of the endoplasmic reticulum chaperone BiP3 regulates XA21-mediated innate immunity in rice. PLoS One, 2010, 5(2): e9262

[29] Wu Q, Hou M, Li L, Liu L, Hou Y, Liu G. Induction of pathogenesis-related proteins in rice bacterial blight resistant gene XA21-mediated interactions with Xanthomonas oryzae pv. oryzae. J Plant Pathol, 2011, 93: 455-459

[30] Hou M, Xu W, Bai H, Liu Y, Li L, Liu L, Liu B, Liu G. Characteristic expression of rice pathogenesis-related proteins in rice leaves during interactions with Xanthomonas oryzae pv. oryzae. Plant Cell Rep, 2012, 31: 895-904

[31] 缪刘杨, 李莉云, 刘钊, 刘雨萌, 江光怀, 杨凤环, 何晨阳, 刘 国振. 五个 WRKY 转录因子在水稻叶片生长和抗病反应中的 表达研究. 生物化学与生物物理进展, 2013, 40: 356-364

Miao L Y, Li L Y, Liu Z, Liu Y M, Jiang G H, Yang F H, He C Y, Liu G Z, Jiang G H. Characteristic expression analysis of five WRKY transcriptional factors in rice leaf growth and disease resistance reaction. Prog Biochem Biophys, 2013, 40: 356-364 (in Chinese with English abstract)

[32] 史佳楠, 李莉云, 徐文静, 关明俐, 李雪姣, 牛东东, 兰金苹, 寀世娟, 刘丽娟, 刘国振. 八个 WRKY 转录因子在水稻叶片 生长和抗病过程中的表达研究. 植物病理学报, 2014(已接受)

Shi J N, Li L Y, Xu W J, Guan M L, Li X J, Niu D D, Lan J P, Dou S J, Liu L J, Liu G Z. Expression analysis of eight WRKY transcription factors in rice leaf growth and disease resistance response. Acta Phytopathol Sin, 2014 (accepted) (in Chinese with English abstract)

[33] 窦世娟, 关明俐, 李莉云, 刘国振. 水稻的病程相关基因. 科 学通报, 2013, DOI: 10.1360/972012-1831

Dou S J, Guan M L, Li L Y, Liu G Z. The pathogenesis-related genes of rice. Chin Sci Bull, 2013, DOI: 10.1360/972012-1831 (in Chinese with English abstract)

[34] Li X, Bai H, Wang X, Li L, Cao Y, Wei J, Liu Y, Liu L, Gong X, $\mathrm{Wu} \mathrm{L}$. Identification and validation of rice reference proteins for western blotting. $J$ Exp Bot, 2011, 62: 4763-4772

[35] 刘国振, 刘斯奇, 吴琳, 徐宁志. 基于抗体的水稻蛋白质组学: 开端与展望. 中国科学: 生命科学, 2011, 41: 173-177 Liu G Z, Liu S Q, Wu L, Xu N Z. Antibody-based rice proteomics: the beginning and perspectives. Sci Sin Vitae, 2011, 41: 173-177 (in Chinese with English abstract) 